\section{$\underset{\substack{\text { hommes } \\ \text { \& migrations }}}{ }$}

Hommes \& migrations

Revue française de référence sur les dynamiques

migratoires

$1301 \mid 2013$

Migrations et mondes ruraux

\title{
Les allées et venues des familles de travailleurs
}

\section{ruraux}

Région centrale de l'État de São Paulo, Brésil

Lidiane Maciel

\section{OpenEdition}

Journals

\section{Édition électronique}

URL : http://journals.openedition.org/hommesmigrations/1902

DOI : 10.4000/hommesmigrations.1902

ISSN : 2262-3353

Éditeur

Musée national de l'histoire de l'immigration

\section{Édition imprimée}

Date de publication : 1 janvier 2013

Pagination : 29-35

ISBN : 978-2-919040-21-6

ISSN : 1142-852X

Référence électronique

Lidiane Maciel, «Les allées et venues des familles de travailleurs ruraux », Hommes \& migrations [En ligne], 1301 | 2013, mis en ligne le 31 décembre 2015, consulté le 01 mai 2019. URL : http://

journals.openedition.org/hommesmigrations/1902; DOI : 10.4000/hommesmigrations.1902 


\section{LES ALLÉES ET VENUES DES FAMILLES DE TRAVAILLEURS RURAUX RÉGION CENTRALE DE L'ÉTAT DE SÃO PAULO, BRÉSIL}

par LIDIANE MACIEL, docteur en sociologie de l'université d'État de Campinas (Unicamp), São Paulo (Brésil)1

La région centrale de l'État de São Paulo attire les migrants venant travailler dans l'agro-industrie. Ces migrants, des petits agriculteurs du nord-est du Brésil, restent le temps des récoltes de la canne à sucre ou des oranges dans la périphérie des grandes villes. Ils partagent ainsi leur existence entre plusieurs espaces. Leur désir est à l'image de leur identité multiple: la recherche paradoxale d'une mobilité stable leur permettant à la fois de conserver leur activité agricole et de s'intégrer là où ils travaillent une partie de l'année.

\section{La région centrale de l'État de São Paulo, dans laquelle ces migrants ont commencé à arriver, a été profondément modifiée par le type d'agriculture développé au cours des dernières années.}

La migration rurale en direction des villes ou entre les villes constitue un processus social en cours dans la société brésilienne. L'État de São Paulo, grâce à son dynamisme économique et social, continue d'être un espace où se développent de nombreux échanges migratoires. Dans les années 1980 et 2000, l'État de São Paulo a connu un changement significatif dans la répartition de sa population. L'intérieur de São Paulo a tendance à gagner une population provenant d'au moins deux flux migratoires différents : le premier au départ de la capitale, le second créé par les travailleurs provenant d'autres États tels que le Paraná, Minas Gerais, Bahia, Pernambuco, Piauí, Maranhão, etc.
La région centrale de l'État de São Paulo, dans laquelle ces migrants ont commencé à arriver, a été profondément modifiée par le type d'agriculture développé au cours des dernières années. Les principales caractéristiques de cette agriculture sont la mécanisation, l'utilisation de pesticides agricoles, le lien avec le marché international et l'exploitation de travailleurs peu qualifiés. C'est dans ce contexte économique que les réseaux familiaux et de travail ont attiré les pauvres du nord-est des États brésiliens de la région. Ces dernières années, les flux migratoires qui ont atteint la région centrale de São Paulo ont commencé à recevoir les populations issues de deux États brésiliens : Piauí et Maranhão, 
États qui ne faisaient pas partie de la cartographie de la migration dans l'État de São Paulo. La région de Matão est l'un des espaces sociaux qui a changé à cause de ces nouveaux flux migratoires. Cette région se démarque dans les années 1990 et 2000 en raison de sa production de jus d'orange, qui nécessite une main-d'œuvre importante, puisque la récolte des oranges est totalement manuelle.

Lorigine de ces travailleurs est diverse, mais on peut remarquer ces dernières années que la récolte des oranges dans la région de Matão a été réalisée en grande partie par des travailleurs migrants. Beaucoup sont de petits agriculteurs du nord-est du Brésil qui, compte tenu des conditions sociales, politiques ou climatologiques de cette région, sont obligés de migrer à la recherche d'emplois temporaires leur garantissant d'avoir les moyens de subvenir aux besoins de leurs fa-

Ces dernières années, milles qui, souvent, restent les flux migratoires qui ont atteint la région centrale de São Paulo ont commencé à recevoir les populations issues de Piauí et Maranhão,

États qui ne faisaient pas partie de la cartographie de la migration dans l'État de São Paulo. dans le nord du Brésil. Cela peut être observé chez les petits agriculteurs de la région de Jaicós, dans le centre-sud de l'État de Piauí.Ces individus vivent une double existence, employés de l'agro-industrie et demeurant à la périphérie des grandes villes pendant une période de l’année, et petits agriculteurs des zones rurales de plusieurs petites villes durant l'autre. Ils ne vivent ni dans le milieu rural ni dans le milieu urbain, mais bien entre ces deux mondes connectés par la pauvreté et le travail.

Pendant les années 2009, 2011, 2012 furent réalisés 40 entretiens qualitatifs de travailleurs ruraux des villes de la région centrale de l'État de São Paulo, ainsi que de la ville de Jaícós dans l'État du Piauí. Ces entretiens ont été mis en parallèle avec les données quantitatives obtenues à partir de différentes bases de données provenant de l'Institut de géographie et de statistique du Brésil (IBGE), du Système de l'analyse des données de l'État de São Paulo (SEADE) ou des indicateurs sociaux annuels du ministère brésilien du Travail. L'objectif était de comprendre les trajectoires migratoires des travailleurs migrants ruraux à la recherche de travail et de revenus dans les États de Piauí et de São Paulo.

\section{Une migration temporaire permanente}

La situation des années 1990 a rendu nécessaires de nouveaux apports théoriques pour l'étude des migrations. L'approche théorique utilisée jusqu'à présent ne prenait pas en compte le processus social en cours². "La compréhension des phénomènes urbains, en particulier celle des nouvelles formes de mobilité spatiale de la population, subit des transformations qui, tout en reflétant la restructuration de l'économie, composent une nouvelle mosaïque des interactions sociales $^{3 \prime}$. Ces nouvelles conditions que nous rappelle Rosana Baeninger nous questionnent sur le fait de savoir ce qu'est un migrant dans ce nouveau contexte. Pour Abdalmalek Sayad, l'immigration des migrants algériens vers la France a présenté une double contradiction: "Oscillant selon les circonstances entre létat provisoire qui la définit de droit et la situation durable qui la caractérise de fait ${ }^{4}$.

Pour cet auteur, ce qui définit ces gens comme migrants est le fait d'être essentiellement une maind'œuvre temporaire et en transit. Notion très similaire à celle mise en lumière par Maria Silva ${ }^{5}$ lors de l'étude de la migration temporaire permanente ${ }^{6}$ vécue par les petits agriculteurs du nord et du nord-est du Brésil vers la région de Ribeirão Preto et vers le centre de l'État de São Paulo. Ces travailleurs sont temporairement employés afin de récolter la canne à sucre et les agrumes

\footnotetext{
2. Au Brésil, les interprétations en matière de migrations jusqu'aux années 1990 ont favorisé l'approche du secteur rural urbain. Ce type de migration a été provoqué par des transformations macro-économiques qui se sont développées au cours des soixante dernières années dans ce pays. 3. Rosana Baeninger, “São Paulo e suas migrações no final do século 20", in São Paulo em Perspectiva, vol. 19, $n^{\circ}$ 3, 2005, p. 86. 4. Abdelmalek Sayad, A imigração os paradoxos da alteridade, São Paulo, Editora da USP, 1998, p. 45. 5. Maria Aparecida de Moraes Silva, "Expropriação da terra, violencia e migração: Camponeses maranhenses no corte da cana em São Paulo", in Cadernos Ceru, vol. 19, n 1, São Paulo, 2008, pp. 84-96. 6. Ce type de migration se produit de la même manière chaque année. Le séjour est temporaire, mais la pratique est permanente.
} 
du complexe agro-industriel de São Paulo. Les migrants ont apporté avec eux des signes distinctifs qui les définissent comme appartenant à Paraíba, Piauí, Pernambuco, Minas Gerais, Bahia, Paraná en Matão, ou à l'État de São Paulo.

Dans ce contexte, la notion d'espace de vie ${ }^{7}$ est très importante. L'espace de vie correspondrait à la formation de réseaux de relations et d'événements liés à la vie familiale, économique, politique, culturelle. Ainsi, le passage d'une résidence à une autre n'implique pas toujours un changement dans l'espace de vie. Les migrations partent toujours d'une résidence de base ${ }^{8}$ et le retour migratoire est lié à cet espace.

Ce concept nous aide à réfléchir à l'identité individuelle des familles qui ont effectué, tout au long de leur trajectoire, de nombreux changements de lieux de vie et qui, lorsqu'on interroge leurs membres sur leur lieu d'origine, considèrent l'ensemble des sentiments et des constructions symboliques apparus dans différents espaces autres que celui où ils sont nés.

\section{La construction d'une identité migrante}

Le lieu d'origine, qui n'est pas toujours le lieu de naissance, aide les individus à se construire une identité régionale ou locale. L'identité des travailleurs ruraux n'est pas toujours liée à leur région de naissance, mais à d'autres espaces de vie créés par le processus migratoire de leur famille pendant leur trajectoire. On peut aussi interpréter le processus de mobilité sociale grâce à Alain Tarrius qui considère que c'est dans le "territoire circulatoire" que se compose le réseau de références des migrants, et que c'est là aussi dans le "territoire circulatoire" que se développent la mémoire collective et la pratique d'échange de valeurs spécifiques. Pour cet auteur,

\section{Carte du Brésil}

En particulier des États de São Paulo et Piauí.

La distance entre l'État de Piauí et de l'État de São Paulo est de 2806 kilomètres. CSOURCE : IBGE, 2010.

les trajectoires spatiales, c'est-à-dire les itinéraires, sont des lieux animés de relations sociales dans lesquelles est présent le jeu de la différenciation culturelle. Le territoire, au-delà de l'identité, produit une altérité propre nécessaire aux relations sociales.

Les "Piauís" - migrants de la ville de Jaícos employés pour la récolte des agrumes dans le Matão à São Paulo -, lorsqu'ils sont interviewés, nous révèlent une tentative d'être de là-bas et d'être d'ici, puisqu'ils souhaitent à la fois garder leurs terres agricoles dans l'État de Piauí tout en s'intégrant à la ville de destination migratoire grâce au travail et à une durée de séjour plus longue.

Il est important de noter que le flux de travailleurs ruraux de Jaicós ne se constitue pas unilatéralement. Non seulement ils migrent vers la ville de Matão, mais aussi vers d'autres villes qui ont besoin de main-d'œuvre pour certains travaux manuels ou les récoltes. Les "territoires circulatoires" sont multiples dans ce domaine d'étude. 


\section{Ruralité et processus migratoire entre la région centrale de São Paulo et le sud-est de l'État de Piauí}

Il y a de grandes différences entre la ruralité établie dans la région centrale de São Paulo et celle de l'État de Piauí au sud-est. La première se rapporte en grande partie à un complexe agro-industriel et la seconde est liée à une agriculture familiale de subsistance. Dans les années 1970, les investissements du ministère de l'Agriculture dans l'État de São Paulo ont permis d'y moderniser la production. La région administrative du centre de São Paulo et celle de Ri-
Les agrumes utilisent en grande partie la maind'œuvre des migrants et, contrairement à la canne

à sucre, dans certaines

régions, on trouve assez souvent des familles entières récoltant des oranges. beirão Preto plus récemment ont commencé à exceller dans la production de canne à sucre destinée à la fabrication d'éthanol et dans la production de jus d'orange, ciblant le marché international. Cette redistribution industrielle dans l'État de São Paulo s'est accompagnée du renforcement du réseau urbain et social entre les municipalités des régions. C'est aussi durant cette période que commence la recherche d'une main-d'œuvre disponible pour faire face aux longues heures de travail dans les champs de canne à sucre et dans les orangeraies de cette région.

Parallèlement au développement du système agroindustriel de la canne à sucre au Brésil, l'industrie des agrumes s'insère également dans le mouvement du système mondial visant à créer de nouveaux emplois en milieu rural. Dès ses débuts, le secteur des agrumes, qui s'est développé à l'intérieur de São Paulo, était déjà inscrit dans une dynamique internationale. La production à grande échelle de jus d'orange a toujours été liée à l'exportation aux États-Unis ou en Europe ${ }^{10}$.
Les agrumes utilisent en grande partie la maind'œuvre des migrants et, contrairement à la canne à sucre, dans certaines régions, on trouve assez souvent des familles entières récoltant des oranges. Ces familles sont surnommées "Bóias-Frias", ce qui signifie "travailleurs qui mangent des aliments froids". Ce terme utilisé par la population locale des villes traduit l'état précaire de leur travail. En général, les travailleurs engagés pour la récolte des oranges apportent leur gamelle pour le déjeuner pris sur le lieu de travail sans qu'ils aient la possibilité de le réchauffer. Par conséquent, les travailleurs mangent des aliments froids.

Les travailleurs ruraux migrants se reconnaissent mal dans la catégorie "Bóias-Frias". Ils se sentent souvent offensés par ce terme qui exprime pour eux l'exclusion sociale. La population locale ne comprend pas la soumission à ce genre d'exploitation, ni les conditions de travail dans les zones rurales. Mais, en essayant d'interpréter l'origine de l'expression, on peut considérer que manger un aliment froid se rapproche de l'état naturel des choses. Comme nous pouvons l'interpréter grâce à l'étude de Lévi-Strauss sur la nature et la culture, il y a une certaine déshumanisation à l'œuvre chez le travailleur migrant rural qui vit dans une ville moderne, mais qui se soumet à un type de travail précaire. Un processus d'exclusion sociale est en cours.

\section{Les villes moyennes: des zones de départ}

Au Brésil, comme dans l'État de São Paulo, l'agriculture est liée au marché international et a eu tendance à se moderniser ces dernières décennies. Mais, dans certaines régions, l'économie agricole reste fortement dominée par les techniques agricoles traditionnelles. Les villes du centre-sud de l'État de Piauí, comme Eslesbão Veloso, Jaicós, Padre Marcos, Várzea Queimada, en sont des exemples. L'agriculture familiale au Brésil se caractérise par une petite propriété, une main-d'œuvre 
familiale, la production d'articles pour l'alimentation, des techniques de gestion traditionnelles et une faible utilisation de la technologie. L'agriculture familiale produit environ $60 \%$ de la nourriture consommée par la population brésilienne. La ville de Jaicós, selon le recensement de 2010, possède actuellement une population de 18000 habitants. Environ 53 \% résident dans des zones considérées comme rurales et d'agriculture familiale. C'est de ces zones, appelées par la population "zones d'intérieur”, que partent les migrants vers São Paulo pour aller récolter les oranges. La municipalité de Jaicós, comme celle d'autres régions du nord-est du Brésil, a subi un processus de stagnation économique et a créé, selon Paul Singer, des aires d'évasion démographique ${ }^{11}$. La région de Matão, située dans la partie centrale de l'État de São Paulo, est composée d'une population permanente de 76000 habitants et d'une population flottante d'environ 5000 habitants durant la récolte des oranges, la municipalité ayant une agro-industrie fortement marquée par la production des oranges. C'est dans ce complexe industriel qu'est employée, temporairement, une partie de la population migrante de la ville de Matão.

Ces dernières années, certaines villes de la région du centre, comme Matão, ont commencé à recevoir un grand nombre de migrants. Ces flux ont au moins deux causes : les réseaux familiaux et de recrutement de main-d'œuvre existant entre les villes de l'État de São Paulo et certaines villes du nord-est du Brésil ; les problèmes économiques et structurels - politiques, fonciers et environnementaux - dans le nord du Brésil stimulant encore davantage le départ de population.

Le caractère temporaire du séjour des migrants à Matão se traduit dans de nombreux aspects de leur vie sociale. La plupart du temps, ils sont logés de façon précaire dans des maisons louées dans les quartiers qui hébergent cette population.

La ville de Matão reçoit chaque année environ 3000 travailleurs de la ville de Jaicós destinés à la 
récolte des oranges. Pour les migrants, les allées et venues sont subordonnées à l'idée d'améliorer leur vie dans leur lieu d'origine, ce qui signifie l'investissement dans une propriété, que ce soit afin de produire les mois sans hiver, les travailleurs ruraux de un énorme marché de biens économiques et symboliques. Le financement se fait par des prêts entre parents, la vente d'objets personnels et de produits agricoles. Il y a au moins deux compagnies de bus qui font le trajet entre les villes de Jaícos et Matão. L'une d'elles est illégale, que les migrants préfèrent souvent car le propriétaire jouit d'un certain prestige parmi les migrants. À leur arrivée dans la ville de Matão, face à un marché immobilier saturé, de nombreux migrants n'ont d'autre choix qu'un habitat précaire. Les propriétaires font payer les loyers par personne ou par famille. Pour eux, la durée des récoltes est une période très lucrative. Dans la ville de Jaicós, migrer quand il n'y a pas d'hiver (une période sans pluies) est un fait qui touche les petits agriculteurs, et de nombreuses familles qui ont des parents dans d'autres villes ou qui ont déjà migré. Les principales cultures produites par cette population sont liées à l'agriculture de subsistance. De nombreux travailleurs agricoles ne sont pas propriétaires de la terre sur laquelle ils travaillent. Par conséquent, ils louent les terres à d'autres propriétaires. La productivité génère peu d'excédent, parce que ces petits agriculteurs sont situés sur les plus mauvaises terres de la ville.

Entre les mois de novembre et de janvier sont plantés divers types de haricots, du manioc, et d'autres fruits comme la noix de cajou. Le travail effectué est familier, les membres de la famille sont responsables de la culture, de la récolte et de la commercialisation. Ce sont les femmes, les enfants et les autres membres de la famille, qui n'ont pas migré pour la récolte des oranges à São Paulo qui se chargent de la récolte des produits de l'agriculture locale. Dans la ville de Matão, ces travailleurs sont employés à la récolte des agrumes entre les mois de juin à décembre ; le travail exige beaucoup d'agilité physique, ainsi le profil type de ces migrants est donc très jeune. Le rythme de la journée de travail 
est imposé par la nécessité de maintenir la productivité. En raison des longues heures de travail en contact avec divers pesticides, de la difficulté d'accès au système de santé de la ville de Matão, d'une alimentation pauvre et d'un logement précaire, de nombreuwx travailleurs migrants tombent malades au début de la récolte et sont obligés de retourner dans leur ville natale.

\section{La force des liens avec le lieu d'origine}

Même si les travailleurs connaissent de mauvaises conditions de vie durant les récoltes des agrumes, c'est ce travail effectué dans les villes qui permet de maintenir leur famille dans le lieu d'origine. C'est grâce à leurs gains qu'ils peuvent augmenter la production agricole dans leur propriété et même acheter une propriété rurale et construire une maison en ville. Le retour en décembre, après la récolte des agrumes dans la ville de Matão, est marqué par l'ouverture des maisons qui ont été fermées lorsque la famille a migré.

Dans la ville de Jaicós, on dit que les migrants qui se rendent à Matão dans l'État de São Paulo "vivent dans le monde". Certaines des personnes interrogées comme Cecilia (45 ans), José (66 ans) et Maria do Socorro (56 ans) renforcent l'idée que ce sont des survivants du processus d'expropriation socio-économique qui affecte la ville. Ce sont des survivants parce qu'ils n'ont jamais eu à vivre dans un monde qui leur est inconnu. Comme le soulignait déjà Klaas Woortman ${ }^{13}$, la migration a une signification symbolique : c'est un rituel qui renvoie les individus qui ont migré vers leur société d'origine avec un statut différent de ce qu'ils avaient auparavant. Ainsi, le phénomène de la migration est responsable de la redéfinition des rôles sociaux entre ceux qui partent et ceux qui restent, en réorganisant les espaces de vie.
Carte de l'État de São Paulo

En évidence la capitale de São Paulo et la ville de Matão SOURCE : Ibg (MARQues AJoutéEs PAR L' AUteur).

\section{Conclusion}

Les migrants de l'État de Piauí dans la région de Matão sont conditionnés à vivre une vie partiellement fragmentée dans le temps et dans l'espace, entre leurs lieux d'origine et de destination. La plupart des revenus sont obtenus à São Paulo, mais l'espace de vie, familial et social, est lié à la communauté rurale dans l'État de Piauí. Le désir d'améliorer leur vie incite ces migrants à quitter leur foyer dans le Nord-Est et à entreprendre un long voyage de plus 3000 kilomètres à la recherche de travail et de revenus. L'espace géographique qui sépare l'État de São Paulo de l'État de Piauí est relié par les allées et venues des migrants.

Cette étude sur les travailleurs migrants à l'intérieur de São Paulo montre clairement les asymétries économiques et sociales du Brésil contemporain. Contrairement à ce qui s'est passé au cours des soixante dernières années où la migration interne avait comme direction les régions métropolitaines, il est important que les chercheurs d'aujourd'hui fassent plus attention aux villes petites et moyennes car, dans les années qui viennent, cellesci vont jouer un rôle important dans la distribution de la population au Brésil. I 